\title{
Multistability, phase diagrams, and intransitivity in the Lorenz-84 low-order atmospheric circulation model
}

Cite as: Chaos 18, 033121 (2008); https://doi.org/10.1063/1.2953589

Submitted: 31 October 2007 . Accepted: 12 June 2008 . Published Online: 25 August 2008

Joana G. Freire, Cristian Bonatto, Carlos C. DaCamara, and Jason A. C. Gallas

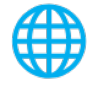

\section{ARTICLES YOU MAY BE INTERESTED IN}

Self-organization of pulsing and bursting in a $\mathrm{CO}_{2}$ laser with opto-electronic feedback Chaos: An Interdisciplinary Journal of Nonlinear Science 25, 097607 (2015); https:// doi.org/10.1063/1.4916923

Relative abundance and structure of chaotic behavior: The nonpolynomial BelousovZhabotinsky reaction kinetics

The Journal of Chemical Physics 131, 044105 (2009); https://doi.org/10.1063/1.3168400

The Hindmarsh-Rose neuron model: Bifurcation analysis and piecewise-linear approximations

Chaos: An Interdisciplinary Journal of Nonlinear Science 18, 033128 (2008); https:// doi.org/10.1063/1.2975967

\section{Seilight Highlights of the best new research} in the physical sciences 


\title{
Multistability, phase diagrams, and intransitivity in the Lorenz-84 low-order atmospheric circulation model
}

\author{
Joana G. Freire, ${ }^{1,2}$ Cristian Bonatto, ${ }^{1}$ Carlos C. DaCamara, ${ }^{2,3}$ and Jason A. C. Gallas ${ }^{1}$ \\ ${ }^{1}$ Instituto de Física, Universidade Federal do Rio Grande do Sul, 91501-970 Porto Alegre, Brazil \\ ${ }^{2}$ Departamento de Física, Faculdade de Ciências, Universidade de Lisboa, 1749-016 Lisboa, Portugal \\ ${ }^{3}$ Centro de Geofísica da Universidade de Lisboa, 1749-016 Lisboa, Portugal
}

(Received 31 October 2007; accepted 12 June 2008; published online 25 August 2008)

\begin{abstract}
We report phase diagrams detailing the intransitivity observed in the climate scenarios supported by a prototype atmospheric general circulation model, namely, the Lorenz-84 low-order model. So far, this model was known to have a pair of coexisting climates described originally by Lorenz. Bifurcation analysis allows the identification of a remarkably wide parameter region where up to four climates coexist simultaneously. In this region the dynamical behavior depends crucially on subtle and minute tuning of the model parameters. This strong parameter sensitivity makes the Lorenz- 84 model a promising candidate of testing ground to validate techniques of assessing the sensitivity of low-order models to perturbations of parameters. (c) 2008 American Institute of Physics.
\end{abstract}

[DOI: $10.1063 / 1.2953589]$

The reliability of simulated climates depends critically on the quality of model parameters. Extensive investigation of parameter space is, however, prohibitively expensive for realistic atmospheric general circulation models which normally involve sets with several thousands of coupled differential equations. The aim of this paper is to report phase diagrams detailing the multistability as observed in the climate scenarios supported by a prototype atmospheric general circulation model, namely, the Lorenz-84 low-order model. As pointed out by Smith, ${ }^{1}$ although it is unreasonable to expect solutions to lowdimensional problems to generalize to a million dimensional spaces, so too it is unlikely that problems identified in the simplified models will vanish in operational models. On the other hand, recent results cited below indicate the possibility that high-dimensional models may behave in a smooth way with respect to changes in parameter values. Our reanalysis of the parameter space uncovers the existence of a remarkably wide new phase where up to four climates coexist simultaneously. Thus, in addition to the familiar sensitive dependence on initial conditions, the final climate (attractor) may depend crucially on subtle and minute tuning of parameters. This new phase is a good testing ground to validate techniques of assessing the sensitivity of a much used low-order model to perturbations of parameters. Our investigation also reveals the existence and inner structuring of wide chaotic phases in Lorenz's flow. Although chaotic phases of discrete mappings have been explored for a number of years now, the exploration of chaotic phases in flows is just starting and certainly demands much more work.

\section{INTRODUCTION}

It is well-established that one essential ingredient controlling the reliability of simulated future climate scenarios is the precision of model parameters. The precision of model parameters is also a determinant factor that greatly shapes the outcome of any model simulation. Dependence on parameters is so important for climate prediction that, for example, an intergovernmental panel on climate change has issued an explicit call for a systematic evaluation of the effect of parameter uncertainties on the simulation of the present climate. ${ }^{2,3}$

Ideally, what one would like to do to assess the reliability of simulated climate is to change model parameters and repeat simulations, validating then the results. This procedure is, however, prohibitively expensive for realistic atmospheric general circulation models (AGCMs) which may involve simulating sets with several thousands of coupled differential equations. ${ }^{4}$ A promising approach to mitigate this severe computational problem is the ingenious idea behind the climateprediction.net, namely, large scale distributed computations exploiting idle processing capacity on personal computers volunteered by the general public around the world. ${ }^{5}$

In order to describe accurately the instantaneous state of the Earth's entire atmosphere, a very large number (of the order of $10^{7}$ ) of variables has to be used. ${ }^{6}$ Such descriptions are perhaps the ones consuming the largest fraction of computational power ever. And this is likely to remain, independently of how powerful computers might become in the future.

Alternatively, a number of questions and processes involved in climate prediction may be conveniently addressed using a considerably simpler approach based in the so-called low-order models, which involve of the order of 100 equations or even less than that. As pointed out by Smith, "although it is unreasonable to expect solutions to lowdimensional problems to generalize to a million dimensional spaces, so too it is unlikely that problems identified in the simplified models will vanish in operational models." It is equally important to note recent results which indicate the possibility that high-dimensional models may behave in a 
smooth way with respect to changes in parameter values. ${ }^{7-9}$ Thus, low-order models may well have little to do with higher-dimensional operational models. But this fact does not detract from their utility in providing useful insight.

A very appealing low-order model of atmospheric circulation is one introduced by Lorenz in 1984, involving just three first-order differential equations, Eqs. (1)-(3) below. As pointed out by Lorenz, his model is perhaps "the simplest possible general circulation model."10,11 Lorenz's model allows one to address specific questions concerning key applications. For instance, how the coexistence of two possible climates combined with variations of the solar heating may give rise to seasons with interannual variability, ${ }^{10-13}$ how the asymmetry between oceans and continents is basic for the system to exhibit complex behaviors, ${ }^{14,15}$ how the climate is affected by the interactions between atmosphere and the oceans, ${ }^{16,17}$ as a test-ground for techniques devised to characterize and measure predictability. ${ }^{18}$ Aside from practical applications, the low-order model has also attracted attention because of certain interesting and subtle mathematical aspects of its differential equations and bifurcational phenomena. ${ }^{19-21}$

While intransitivity, i.e., coexistence of attractors ("multistability"), in the Lorenz-84 model has been known and studied since the original publications of Lorenz, ${ }^{10,11}$ so far the relative abundance of the different climates (attractors) of the model was not yet considered. The main emphasis so far has been mostly on describing the rich variety of dynamical behaviors and bifurcations and on the manifold mathematical subtleties of the equations of motion. ${ }^{19-21}$ Our goal here is to investigate the prevalence of all possible climates supported by the Lorenz-84 model. To this end, we compute numerical phase diagrams of each climate, based on the Lyapunov spectrum. We wish to obtain a quantitative description of the parameter space which is detailed enough to allow subsequent investigation of the influence in ensemble statistics of some highly intransitive parameter domains described below. The low-order model is obviously too simplified to represent full AGCM. But Lorenz's model has the virtue of allowing its parameter and phase spaces to be sampled exhaustively; a feature that we exploit here.

Apart from intransitivity diagrams, there is an additional twist that we wish to exploit. The substantial increase in computer power opens the way to explore long overdue challenges: the characterization of chaotic phases of flows. While the structuring of chaotic phases in maps is fairly well understood, at least in low dimensions, the equivalent problem for systems ruled by sets of nonlinear ordinary differential equations remains essentially open. An enticing question is that concerning the interconnections among networks of infinite regular phases which exist abundantly embedded in chaotic phases.

\section{LORENZ-84 LOW-ORDER MODEL}

A comprehensive review of the Lorenz-84 model is given in Sec. 9.3 of the nice book of Tel and Gruiz. ${ }^{6}$ The model is defined by three nonlinear autonomous differential equations, namely, ${ }^{10,11}$

$$
\begin{aligned}
& \dot{x}=-y^{2}-z^{2}-a x+a F, \\
& \dot{y}=x y-y-b x z+G, \\
& \dot{z}=b x y+x z-z .
\end{aligned}
$$

Here, $x$ represents the intensity of the symmetric globeencircling westerly wind current, and also the poleward temperature gradient, which is assumed to be in permanent equilibrium with it. The variables $y$ and $z$ represent the cosine and sine phases of a chain of superposed large-scale eddies, which transport heat poleward at a rate proportional to the square of their amplitude, and transport no angular momentum at all.

The nonlinear contributions $x y$ and $x z$ in Eqs. (2) and (3) represent amplification of the eddies through interaction with the westerly current; this occurs at the expense of the westerly current, as indicated by the terms $-y^{2}$ and $-z^{2}$ in Eq. (1). The variables have been scaled so that the coefficients are unity. The terms $-b x z$ and $b x y$ represent displacement of the eddies by the westerly current, and the coefficient $b$, greater than unity, allows the amplification. The linear terms represent mechanical and thermal damping; the damping time for the eddies has been chosen as the time unit, while the coefficient $a$, if less than unity, allows the westerly current to damp less rapidly than the eddies. So, only the regions where $a<1$ and $b>1$ are investigated. The constant terms $a F$ and $G$ in the model represent symmetric and asymmetric thermal forcings; $F$ and $G$ are the values to which $x$ and $y$ would be driven if the westerly current and the eddies were not coupled. Lorenz identifies the eddies with Rossby waves, even though a prominent mechanism for wave propagation identified by Rossby ${ }^{22}$ is missing from the model. Note that since the model is invariant under the transformation $(x, y, z, G)=(x,-y,-z,-G)$, there is a symmetry relative to the $G=0$ axis so that it is enough to investigate the region $G \geqslant 0$.

For different intensities of the axially symmetric and asymmetric thermal forcing Lorenz ${ }^{10,11}$ found the model to support (i) one or two stable steady-state solutions, (ii) one or two stable periodic solutions, or (iii) irregular (aperiodic) solutions. In other words, for a fixed set of parameters one finds intransitivity involving two distinct attractors, each one consisting of two disjoint pieces and corresponding to a closed curve, and associated with two different climates.

Figure 1 shows representative examples of the timeseries originally studied by Lorenz, illustrating the onset of intransitivity as the asymmetrical thermal forcing $G$ increases from $G=0.2$ to 0.8 . For $G=0.2$, all initial conditions eventually converge to the very regular oscillations seen on the leftmost panel of the figure. In contrast, for $G=0.8$, the attractor existing originally in Fig. 1(a) evolves into the one shown in Fig. 1(b) and a new coexisting periodic solution, shown in Fig. 1(c), appears. In other words, the phase-space contains two distinct basin of attraction when $G=0.8$, instead of the single basin existing for $G=0.2$. 

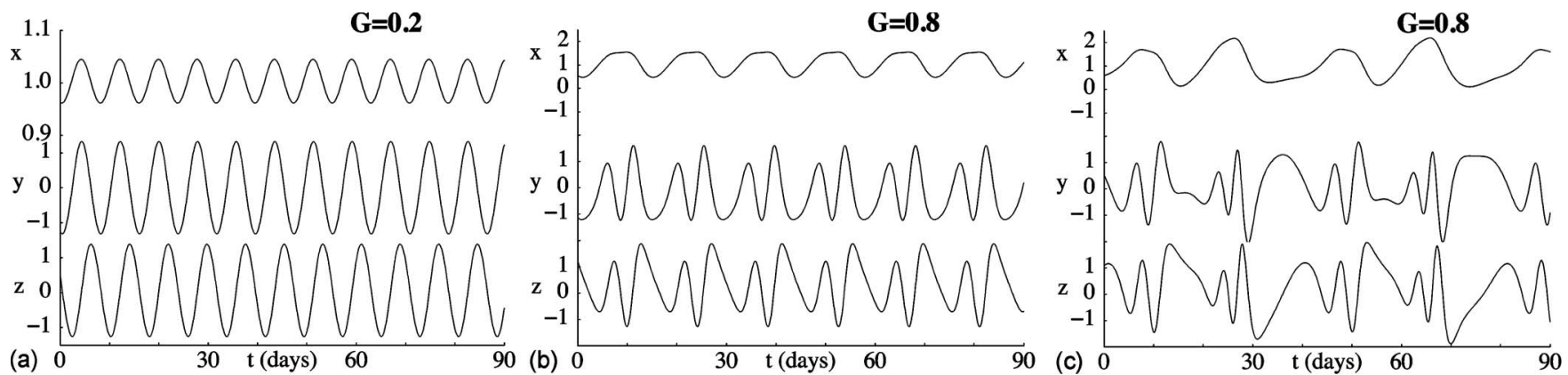

FIG. 1. The onset of intransitivity as $G$ increases. (a) For $G=0.2$ there is a single periodic solution, illustrated here when starting from ( $x, y, z)$ $=(0.5,-1.1,1.0)$. For $G=0.8$ there are two distinct solutions: (b) periodic solution when starting from the same initial condition; (c) additional periodic solutions obtained when starting from $(0.7,-0.4,0.6)$. In all panels: $F=8, a=0.25$, and $b=4$.

\section{FOUR COEXISTING ATTRACTORS}

Figure 1 illustrates the pair of coexisting attractors found by Lorenz. The purpose of this section is to show that for parameters like, for example,

$$
P \equiv(F, G, a, b)=(6.846,1.287,0.25,4),
$$

four stable attractors coexist in phase space (see Fig. 6 below). The model has already been known as a rather nontrivial low-order system. With this finding it becomes an even more complex, useful tool for investigating problems of climate dynamics in a simple setting. The coexistence of a relatively high number of attractors is representative of what we find over extended parameter regions, as discussed in the next section.

The simplest attractor found for the parameters defining point $P$ in Eq. (4) is a fixed point located near

$$
\Omega \equiv(x, y, z)=(0.01746001,0.3032854,0.0926390) .
$$

In addition to this fixed point there are also three distinct time-dependent solutions as shown in Figs. 2 and 3 in two different representations. In these figures the labels A, B, C, D are used to mark the four coexisting attractors.

Although the attractors in Figs. 3(a) and 3(b) look somewhat similar, the splitting seen in Fig. 3(b) indicates clearly that their periods involve a distinct number of peaks. For instance, while attractor A has two peaks that repeat periodi- cally, attractor B has four peaks doing so. That the repetitions occur in this way is difficult to recognized from Fig. 2, due to the scales involved.

After realizing the coexistence of four attractors at $\mathrm{P}$, it is natural to inquire about the relative probability of finding each individual attractor in phase-space, as a function of the initial conditions. The answer may be found numerically by computing the individual basins of attraction of each solution for suitably chosen windows.

To this end we constructed histograms based on Lyapunov exponents ${ }^{23-30}$ computed for Eqs. (1)-(3), for a rectangular mesh of initial conditions covering a window in the $x \times y$ plane. The Lyapunov spectra was determined using a fourth-order Runge-Kutta integrator with a fixed step size of 0.01 units, corresponding physically to $1.2 \mathrm{~h}$. Typically, a transient of $7 \times 10^{4}$ time steps is first removed, with exponents then computed during a time interval 20 times larger than the transient, since close to bifurcations it takes considerably more time for the Lyapunov exponent to settle, a feature familiar in the computation of exponents.

Figure 4 shows two examples of histograms computed for the parameter point $\mathrm{P}$ of Eq. (4). In this figure the letters A, B, C, D mark peaks corresponding to attractors with similar labels in Figs. 2 and 3. The histograms in Fig. 4 were computed for two different regions and resolutions in phase space. Figure 4(a) was computed for a grid of $600 \times 600$ initial conditions, covering the phase-space window shown in Fig. 5(a). In this figure, the relative abundances of attrac-
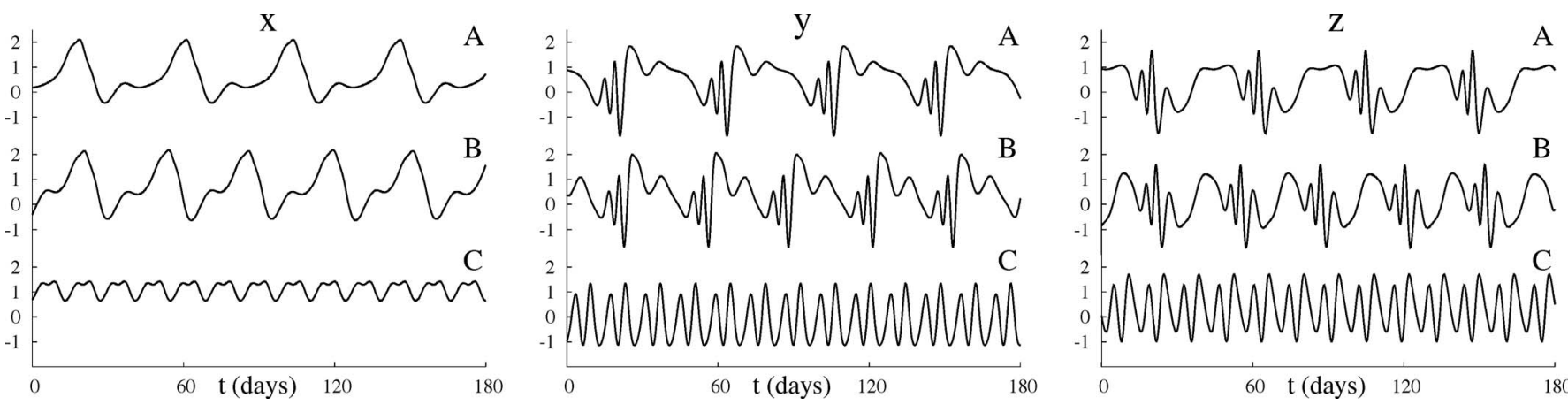

FIG. 2. The three nontrivial climates which coexist with the fixed point $\Omega$ for parameters $P$ given in Eq. (4). Initial conditions for attractors A, B, C are, respectively, $(x, y, z)=(-0.6,-0.58,0),(0.87,-1.4,0)$, and $(0.71,-0.96,0)$. 

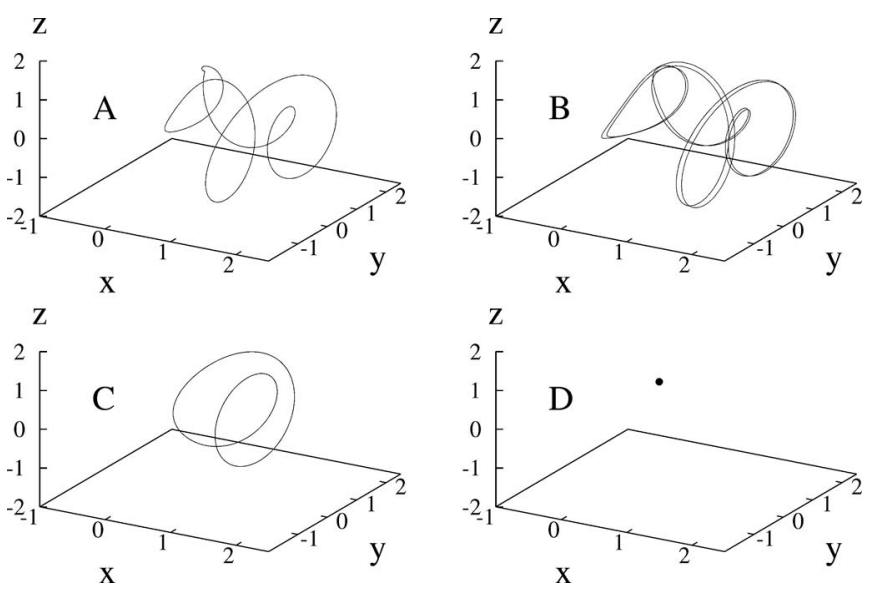

FIG. 3. Three-dimensional views of the four attractors coexisting at the point $P$ defined in Eq. (4) and indicated by the white dot in Fig. 6. The fixed point seen in panel D is located at the point $\Omega$ defined in Eq. (5).

tors A, B, C, D are $35.06 \%, 24.70 \%, 0.23 \%, 40.01 \%$, respectively. In contrast, Fig. 4(b) was obtained for a grid of 750 $\times 750$ initial conditions covering the window shown in Fig. 5(b). The relative abundances of A, B, C, D are $37.12 \%$, $21.00 \%, 2.15 \%, 39.73 \%$, respectively. In both figures, the largest D peak, near -1.2 , corresponds to the fixed point $\Omega$. Comparing the histograms in Figs. 4(a) and 4(b) one recognizes that the relative distribution is not much affected by the distinct regions and distinct resolutions used to obtain them.

Figure 5 shows the very intricate structuring of the basins of attraction for the parameters at point $\mathrm{P}$. The basins were easily plotted with the help of the histograms in Fig. 4. They allow us to discriminate attractors in phase space by coloring in a similar way all exponents that fall under a given peak. For instance, the basins corresponding to the histograms in Figs. 4(a) and 4(b) are shown in Figs. 5(a) and 5(b), respectively. The basins seen in Fig. 5 show $z=0$ sections of the phase space, indicating clearly that the four basins are very intertwined and imply strong final state sensitivity on initial conditions. Figure 5(a) also shows that the basins of the fixed point D and attractors A and B are considerably
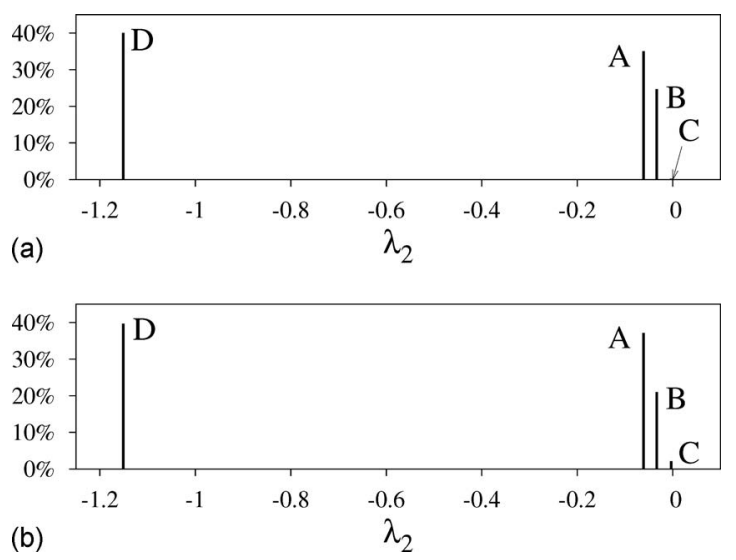

FIG. 4. The relatively invariant volume of the basins of attraction of the four attractors at $\mathrm{P}$, determined by the second largest Lyapunov exponent for two regions and resolutions in phase space. (a) Histogram for $600 \times 600$ initial conditions covering the window shown in Fig. 5(a). (b) Histogram for 750 $\times 750$ initial conditions covering the window shown in Fig. 5(b); see text.
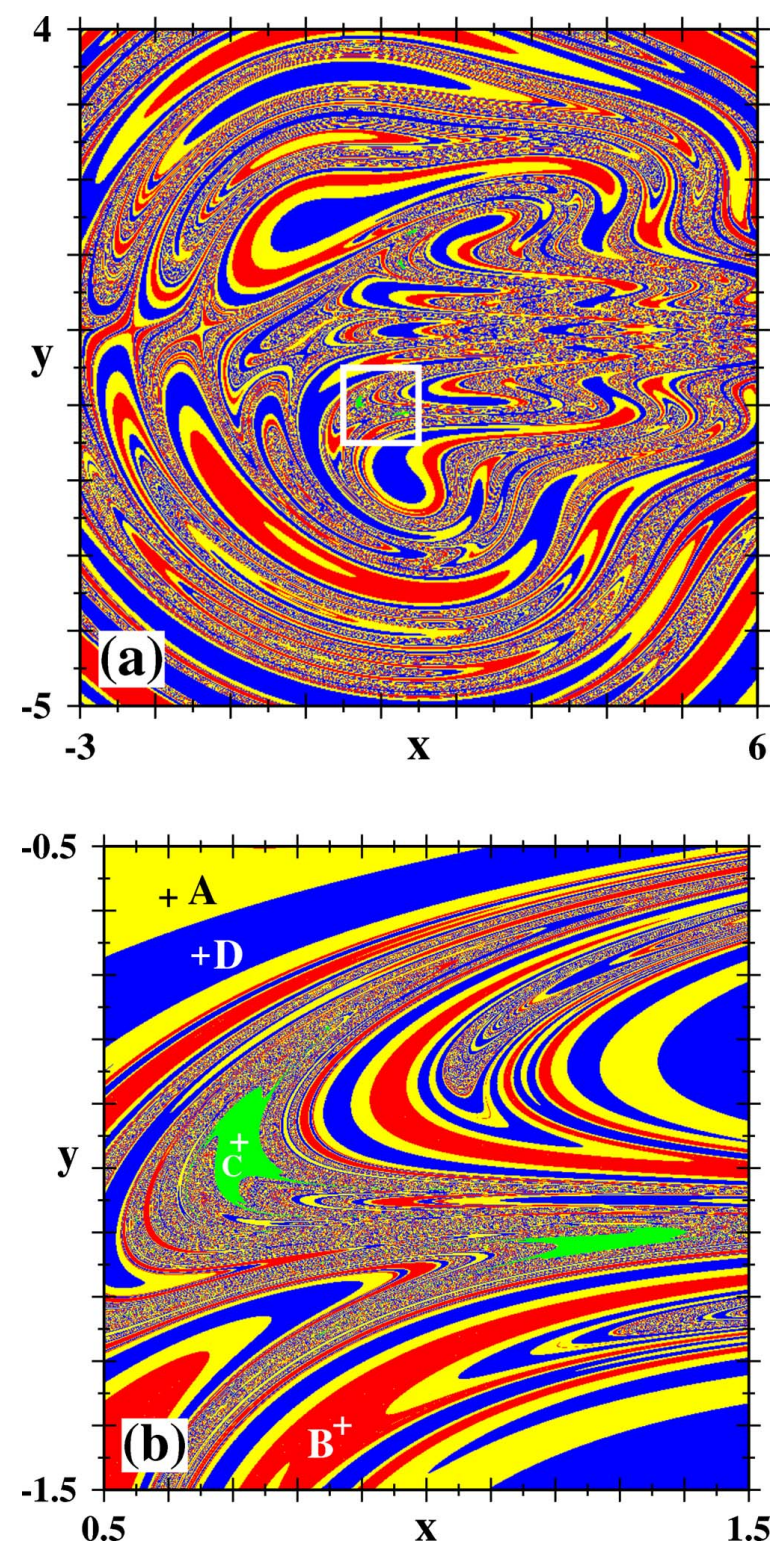

FIG. 5. (Color online) Very strong sensitivity to initial conditions illustrated by basins of attraction for a $z=0$ surface section of Eqs. (1)-(3). The four colors represent the four attractors coexisting for parameters $P$, Eq. (4), colored according to the four peaks in the histograms in Fig. 4. (a) A large window of phase-space; (b) magnification of the box in (a). The letters A, B, C, D mark basins using the same labels in Figs. 2 and 3. The crosses indicate initial conditions $(x, y, z)$ leading to the attractors $\mathrm{A}, \mathrm{B}, \mathrm{C}, \mathrm{D}$, respectively: $(-0.6,-0.58,0),(0.87,-1.4,0),(0.71,-0.96,0),(0.65,-0.67,0)$.

larger than that of attractor C, essentially invisible in this scale, and that may be easily missed under low resolution or washed out in the presence of noise. The basin of the attractor $\mathrm{C}$ is visible in the zoom presented in Fig. 5(b). The basins in Fig. 5 seem to have the Wada property, an indicator of strong unpredictability of the parameters in this phase. ${ }^{31,32}$

\section{INTRANSITIVITY DIAGRAMS}

The purpose of this section is to present phase diagrams discriminating all possible climates of the Lorenz-84 model, and to characterize the abundance of intransitivity, ${ }^{10,11}$ i.e., the abundance of multistability in parameter space. We present phase diagrams showing that the several attractors 


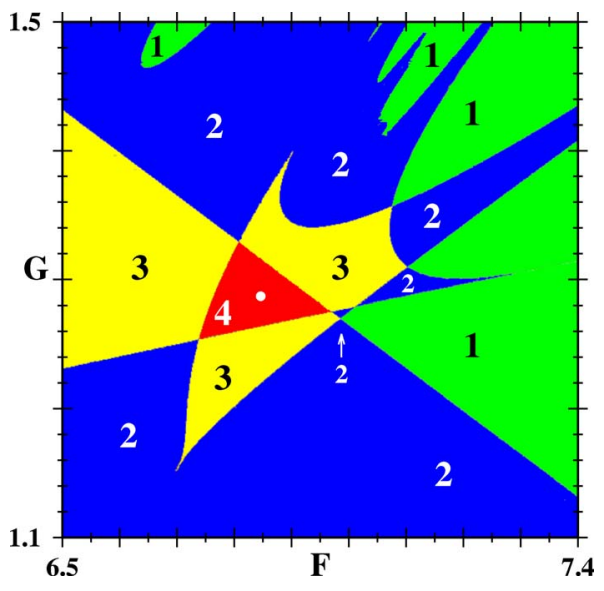

FIG. 6. (Color online) Intransitivity diagram in $F \times G$ space. The numbers indicate the number of distinct climates (attractors) which coexist. The white dot marks the point P of Eq. (4). Here $a=0.25$ and $b=4$.

discussed in the previous section exist over relatively wide regions of parameter space and describe the fourfold "foliated" nature of the parameter space, as induced by the initial conditions leading to individual climates (attractors).

As it is well known, the final attractor toward which the system converges usually depends sensitively on the initial conditions used to start the integration. ${ }^{29,30}$ This is illustrated in Fig. 1 for $G=0.8$. A consequence of this fact is that phase diagrams normally contain "overlaps," i.e., parameter regions where more than one attractor coexist simultaneously. Such overlap of distinct attractors is the same one faced when plotting bifurcation diagrams in the presence of intransitivity.

Figure 6 shows a phase diagram discriminating with colors the number of different attractors coexisting in a 600 $\times 600$ window in parameter space, as indicated by the labels. The shape and volume of the coexistence regions varies considerably. The triangular-shaped region containing the label 4 indicates the domain where four attractors coexist. The white dot roughly at the center of the region indicates the location of the point $\mathrm{P}$ of Eq. (4).

Figure 6 was obtained by determining the boundaries of the parameter planes corresponding to each coexisting attractor. Such boundaries were obtained by following individual attractors in all directions in parameter space, as far as possible.

When performing computations for large sets of parameters and initial conditions one normally tunes integrators to produce reliable exponents by conducting a reasonable number of tests and assuming that such tests define the quality of all subsequent integrations. ${ }^{23,24}$ However, as it is also the case in the computation of bifurcation diagrams, ${ }^{29,30}$ near bifurcation boundaries there is a considerable "numerical lethargy," a pronounced increase in the transient time need to approach the final attractor and to assure convergence of Lyapunov exponents. For these reasons, the boundaries of the region where four different attractors coexist in Fig. 6 are computationally time consuming to define accurately with high resolution. While we believe the overall volume and structuring of the boundaries in Fig. 6 to be essentially cor- rect, small gaps and fluctuations might exist along the boundaries. ${ }^{33-37}$ Therefore, boundaries should be regarded as schematic in the figure. At any rate, our present aim is to show the existence of an extended four-climate phase, not to define unambiguously the structure of the boundaries.

Each of the coexisting attractors coexisting in Fig. 6 has a corresponding basin of attraction in phase space which, when properly scanned, reveals a foliated structuring of the parameter space as displayed in the four panels in Fig. 7. In this, and in similar figures below, we plot $600 \times 600$ Lyapunov exponents on an equally spaced rectangular grid. Gray tonalities are used to represent parameter regions containing fixed points and other periodic motions (i.e., negative exponents). In contrast, yellow and red colors are used to represent chaotic phases (i.e., positive exponents), with red indicating exponents of larger magnitude.

When more than one attractor coexists, we selected one of them to define the color in the figures, usually chosen to maximize the information content of the phase diagram. Colors represent always exponents of largest nontrivial magnitudes, meaning that the second largest exponent was used whenever the magnitude of the largest exponent was found to be zero. Furthermore, although the same scale of colors is used for all pictures, scales were renormalized to reflect the maximum and minimum exponents present in each individual figure (instead of using a fixed color scale for all figures).

Altogether, we find four distinct sets of initial conditions, leading to the four parameter planes shown in Fig. 7. The individual panels in Fig. 7 were computed by starting from a fixed arbitrary initial condition on an arbitrary boundary, here the leftmost boundary, and then proceeding by "following the attractor." 33 By this we mean to follow as much as possible the evolution of that particular attractor found at the initial boundary, by repeating the following expedient: (i) record the value of all variables at the end of an integration for a fixed set of parameters; (ii) increment parameters infinitesimally; (iii) use the recorded values of the variables as initial conditions to start integration for the new (incremented) parameters.

For the record, we mention that Fig. 6 was obtained by combining in a single figure the results contained in the four panels of 7. Figure 6 results from the determination of the number of coexisting climates done by the analysis of the Lyapunov histograms for $4 \times 600 \times 600$ parameter pairs $(F, G)$, a time-consuming numerical task.

Figure 8 presents a larger view of parameter space, obtained by following the attractor found on the left $F=3$ boundary. The roughly triangular-shaped region seen in the upper left corner marks the domain of fixed points investigated by Lorenz. ${ }^{10,11}$ The parameter region contained inside the box is the same considered previously in Figs. 6 and 7. The rough characterization reported by Roebber ${ }^{16}$ agrees well with the classification presented in our Fig. 8. The very coarse-grained classification of Roebber is by far the most detailed classification that we are aware of for the climates of the Lorenz-84 model.

As mentioned in the Introduction, while relatively wide portions of the $F \times G$ parameter plane have been considered 


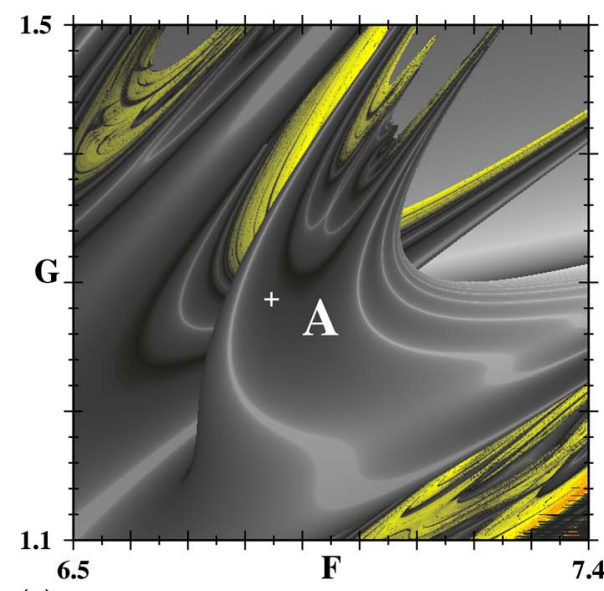

(a)

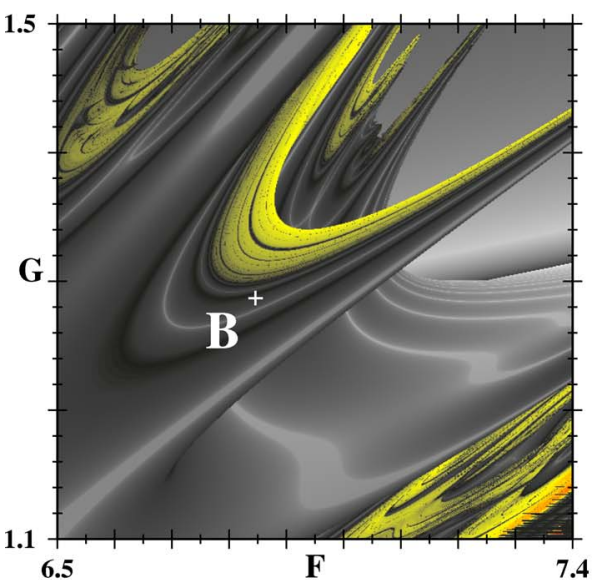

(b)

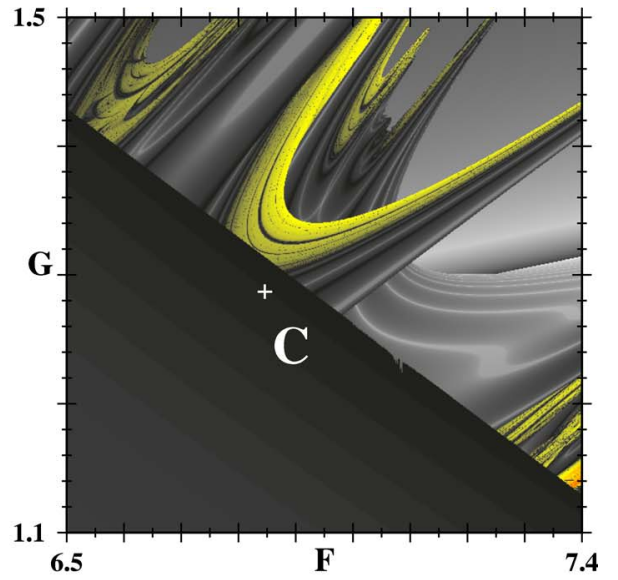

(c)

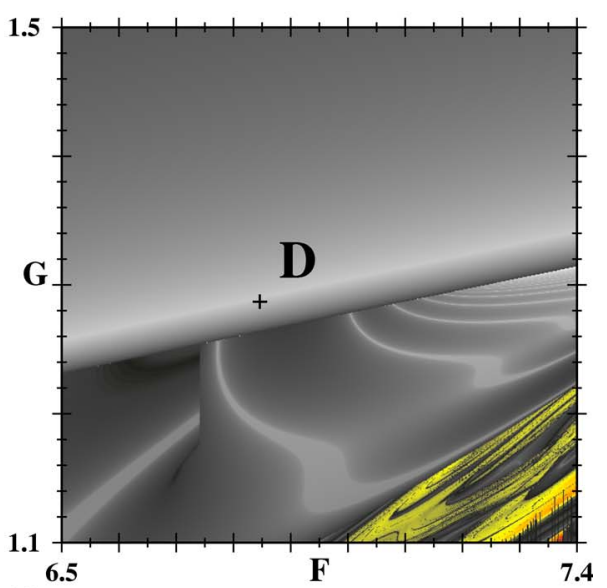

(d)
FIG. 7. (Color online) The four parameter planes A-D which overlap to produce the diagram of the density of attractors depicted in Fig. 6. Here and in similar figures below, fixed points and other periodic solutions (negative exponents) are represented in dark shadings. Chaotic phases (positive exponents) are shown in yellow and red colors (lighter shadings). Crosses mark the point $\mathrm{P}$ of Eq. (4). Here $a$ $=0.25$ and $b=4$.

before by a number of authors, virtually all computations so far were done only for Lorenz's choice of $a=0.25$ and $b=4$. The exception are the considerations of van Veen, ${ }^{21}$ in Sec. 5.1 , who studies the case $a=0.35$ and $b=1.33$. To check what happens when $a$ and $b$ are varied systematically we performed one additional experiment.

Figures 9(a) and 9(b) show phase diagrams when one considers variations of $a$ and $b$ around the values considered originally by Lorenz, while maintaining $F=8$ and $G=1$ fixed.

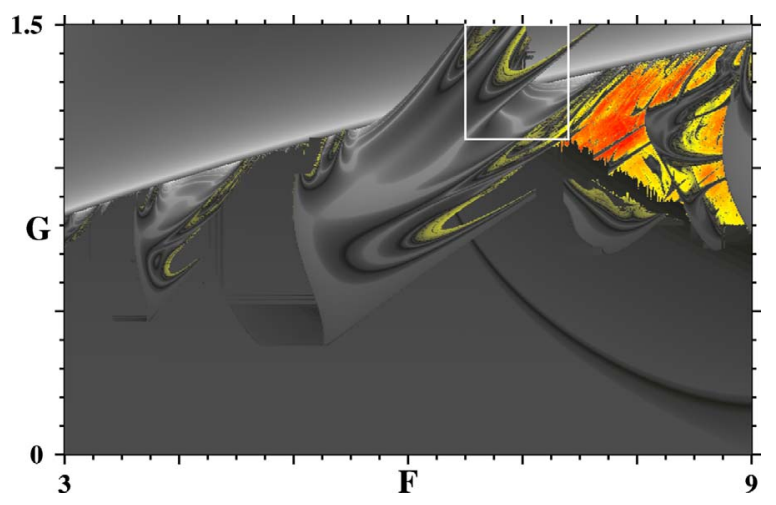

FIG. 8. (Color online) Global view of the $F \times G$ plane discriminating regular phases (shown in dark shadings) and chaotic phases (in color). The chaotic phase is riddled with substructurings and accumulations with characteristic scaling properties (Ref. 35). The box marks the parameter window shown in Figs. 6 and 7. Here $a=0.25$ and $b=4$.
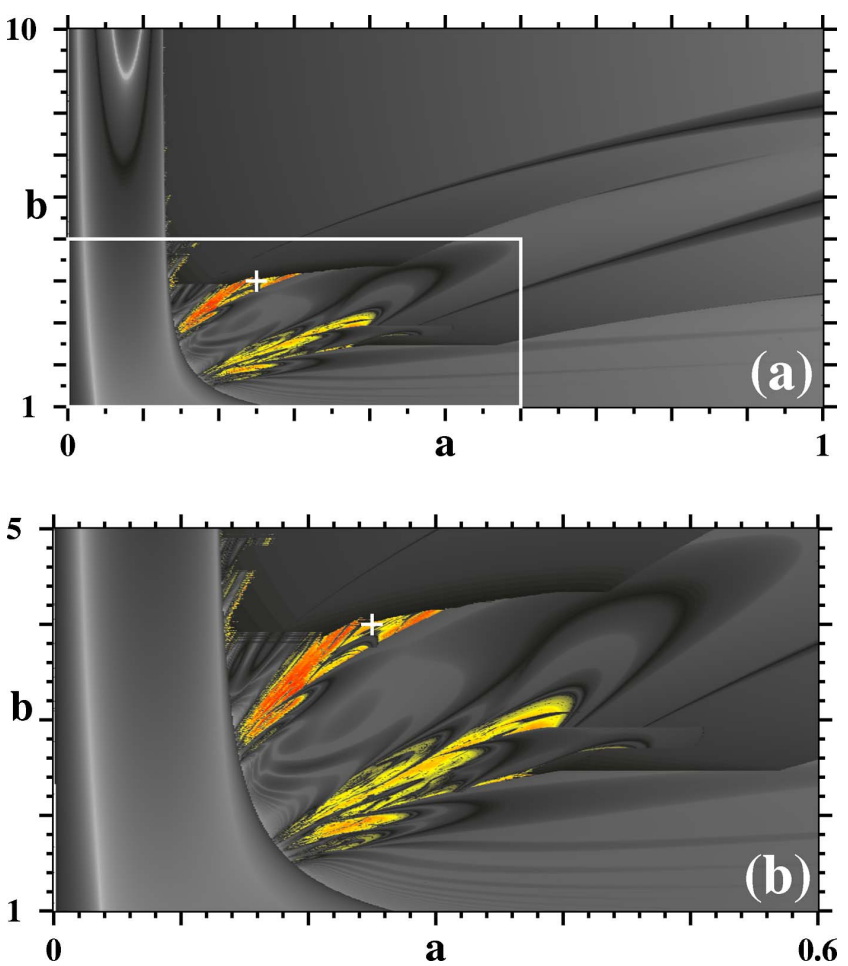

FIG. 9. (Color online) Phase diagrams in $a \times b$ space when fixing $F=8$ and $G=1$. The parameters studied by Lorenz and others are $a=0.25, b=4$, indicated by the cross. (a) Global view, showing predominance of periodic solutions, represented by the dark shadings, proportional to the magnitude of the exponents. (b) Zoom of the box in (a), the region containing chaotic solutions (lighter yellow/red shadings). 
Figures 9(a) and 9(b) were computed in the same way as in Figs. 7 and 8. As seen from Fig. 9(a), periodic behaviors (dark shadings) clearly dominate in this portion of the $a$ $\times b$ plane. Chaotic solutions (in yellow/red lighter shadings) appear however, concentrated on a relatively smaller region, seen in Fig. 9(b), emphasizing that chaos exists for relatively wide variations of $a$ and/or $b$ forcings, mainly for $a$ less than about 0.4. Preliminary work indicates that intransitivity should be also abundant when varying $a$ and $b$ while keeping $F$ and $G$ fixed. This interesting possibility remains, however, to be investigated.

\section{FINAL REMARKS}

We investigated coexistence and parameter dependence of all distinct climate scenarios supported by the Lorenz-84 general circulation model. We based our study on phase diagrams paying particular attention to the number, nature, and relative abundance of the climate scenarios supported by the model. A striking result is that the final climate scenario crucially depends on subtle and minute tuning of parameters. Albeit for a severely truncate low-order model, our work provides evidence corroborating the belief that a proper quantification of the sensitivity of climate models with respect to perturbation of parameters is crucial to assess both the robustness and the significance of the climate scenarios. ${ }^{3}$

An attractive open problem now is the obvious need of performing more realistic numerical experiments when model parameters are subject to both noise and slowly varying external forcings, e.g., the annual cycle of the solar heating via periodic change of $F$. An adequate characterization of the climate scenarios supported by the model may be achieved in this case by applying the ergodic theory of dynamical systems ${ }^{38}$ and the so-called ensemble approach. ${ }^{39,40}$ This approach has been successfully used in several studies, e.g., in assessing the suitability of general circulation models for making seasonal predictions, ${ }^{41}$ in separating the variability of the atmospheric flow into an internal part due to atmospheric dynamics and an external part due to sea surface temperature forcing ${ }^{42}$ and in understanding the role of climate forcings and chaos (unforced variability) in climate. ${ }^{43}$ A study of the climate scenarios supported by the Lorenz-84 model when subjected to periodic forcing is reported elsewhere. $^{44}$

The small quantity of equations of Lorenz-84 model could be explored to build a system of a class that is attracting much attention nowadays, namely, networks of coupled (atmospheric) oscillators. When combined with ideas of graph theory, such coupled oscillators are helpful to investigate the impact of network and subnetwork architectures on global properties, synchronization, and coherence of complex systems in general. ${ }^{45,46}$

\section{ACKNOWLEDGMENTS}

J.G.F. thanks Fundação para a Ciência e a Tecnologia, Portugal, for a Ph.D. Fellowship and for supporting her study stays in Brazil. C.B. thanks a Ph.D. Fellowship of the Con- selho Nacional de Desenvolvimento Científico e Tecnológico (CNPq), Brazil. J.A.C.G. thanks support from CNPq and the Air Force Office of Scientific Research under Contract No. FA9550-07-1-0102.

${ }^{1}$ L. Smith, Proc. Natl. Acad. Sci. U.S.A. 99, 2487 (2002).

${ }^{2}$ Climate Change 2001: The Scientific Basis Contribution of the Working Group I to the Third Assessment Report on the Intergovernmental Panel on Climate Change, edited by T. Houghton, Y. Ding, D. J. Griggs, M. Noguer, P. J. van der Linden, X. Dai, K. Maskell, and C. A. Johnson (Cambridge University Press, Cambridge, 2001), p. 511.

${ }^{3}$ US Climate Change Science Program, 2007: Climate Models: An Assessment of Strengths and Limitations for User Applications.

${ }^{4}$ A. Woods, Medium-Range Weather Prediction: The European Approach, European Centre for Medium-Range Weather Forecasts (ECMWF) (Springer, New York, 2005); J. H. Hack, "Climate system simulation: Basic numerical and computational concepts," in Climate System Modeling, edited by K. E. Trenberth (Cambridge University Press, Cambridge, 1992), pp. 283-318.

${ }^{5}$ D. A. Stainforth, T. Aina, C. Christensen, M. Collins, N. Faull, D. J. Frame, J. A. Kettleborough, S. Knight, A. Martin, J. M. Murphy, C. Piani, D. Sexton, L. A. Smith, R. A. Spicer, A. J. Thorpe, and M. R. Allen, Nature (London) 433, 403 (2005).

${ }^{6} \mathrm{~T}$. Tel and M. Gruiz, Chaotic Dynamics, An Introduction Based on Classical Mechanics (Cambridge University Press, Cambridge, 2006).

${ }^{7}$ D. J. Albers and J. C. Sprott, Nonlinearity 19, 1801 (2006).

${ }^{8}$ D. J. Albers, J. C. Sprott, and J. P. Cruchfield, Phys. Rev. E 74, 057201 (2006).

${ }^{9}$ V. Lucarini, A. Speranza, and R. Vitolo, Physica D 234, 105 (2007).

${ }^{10}$ E. N. Lorenz, Tellus, Ser. A 36A, 98 (1984).

${ }^{11}$ E. N. Lorenz, Tellus, Ser. A 42A, 378 (1990).

${ }^{12}$ R. A. Pielke and X. Zeng, J. Atmos. Sci. 51, 155 (1994).

${ }^{13}$ H. Broer, C. Simó, and R. Vitolo, Nonlinearity 15, 1205 (2002).

${ }^{14}$ C. Masoller, A. Schifino, and L. Romanelli, Phys. Lett. A 167, 185 (1992)

${ }^{15}$ C. Masoller, A. Schifino, and L. Romanelli, Chaos, Solitons Fractals 6, 357 (1995).

${ }^{16}$ P. J. Roebber, Tellus, Ser. A 47A, 473 (1995).

${ }^{17}$ H. W. Broer and R. Vitolo, Discrete Contin. Dyn. Syst., Ser. B 10, 401 (2008)

${ }^{18}$ J. M. González-Miranda, Phys. Lett. A 233, 347 (1997).

${ }^{19}$ A. Shilnikov, G. Nicolis, and C. Nicolis, Int. J. Bifurcation Chaos Appl. Sci. Eng. 5, 1701 (1995).

${ }^{20}$ V. Pelino and A. Pasini, Phys. Lett. A 291, 389 (2001).

${ }^{21}$ L. van Veen, Int. J. Bifurcation Chaos Appl. Sci. Eng. 13, 2117 (2003).

${ }^{22}$ C. G. Rossby, J. Mar. Res. 5, 38 (1939).

${ }^{23}$ J. A. C. Gallas, Appl. Phys. B B60, S-203 (1995), special supplement, festschrift in honor of Herbert Walther.

${ }^{24}$ A. R. Zeni and J. A. C. Gallas, Physica D 89, 71 (1995).

${ }^{25}$ C. Bonatto, J. C. Garreau, and J. A. C. Gallas, Phys. Rev. Lett. 95, 143905 (2005).

${ }^{26}$ C. Bonatto and J. A. C. Gallas, Phys. Rev. E 75, 055204(R) (2007).

${ }^{27}$ C. Bonatto and J. A. C. Gallas, Philos. Trans. R. Soc. London, Ser. A 366, 505 (2008); Phys. Rev. Lett. 101, 054101 (2008).

${ }^{28}$ C. Bonatto, J. A. C. Gallas, and Y. Ueda, Phys. Rev. E 77, 026217 (2008)

${ }^{29}$ J. C. Sprott, Chaos and Time-Series Analysis (Oxford University Press, Oxford, 2003).

${ }^{30}$ R. C. Hilborn, Chaos and Nonlinear Dynamics: An Introduction for Scientists and Engineers, 2nd ed. (Oxford University Press, Oxford, 2000).

${ }^{31}$ H. E. Nusse and J. A. Yorke, Science 271, 1376 (1996).

${ }^{32}$ J. Aguirre and M. A. F. Sanjuan, Physica D 171, 41 (2002).

${ }^{33}$ The structuring of the innermost boundaries might involve oscillations and gaps as discussed in J. A. C. Gallas, C. Grebogi, and J. A. Yorke, Phys. Rev. Lett. 71, 1359 (1993).

${ }^{34}$ H. M. Osinga, Phys. Rev. E 74, 035201(R) (2006); J. Differ. Equations 12, 997 (2006).

${ }^{35}$ J. G. Freire, C. A. Torrico-Chávez, and J. A. C. Gallas, "Structuring of periodic waveforms in chaotic phases of a atmospheric circulation model" (submitted).

${ }^{36}$ J. A. C. Gallas, Phys. Rev. Lett. 70, 2714 (1993).

${ }^{37}$ J. A. C. Gallas, Physica A 202, 196 (1994).

${ }^{38}$ A. Lasota and M. C. Mackey, Chaos, Fractals and Noise: Stochastic Aspects of Dynamics (Springer-Verlag, Berlin, 1994), p. 472. 
${ }^{39}$ C. E. Leith, Mon. Weather Rev. 102, 409 (1974); Nature (London) 276, 352 (1978).

${ }^{40}$ E. S. Epstein, Tellus 21, 739 (1969).

${ }^{41}$ A. Kumar, M. Hoerling, M. Ji, A. Leetmaa, and P. Sardeshmukh, J. Clim. 9, 115 (1996).

${ }^{42}$ A. Harzallah and R. Sadourny, J. Clim. 8, 474 (1995).

${ }^{43}$ J. Hansen, M. Sato, R. Ruedy, A. Lacis, K. Asamoah, K. Beckford, S. Borenstein, E. Brown, B. Cairns, B. Carlson, B. Curran, S. de Castro, L. Druyan, P. Etwarrow, T. Ferede, M. Fox, D. Gaffen, J. Glascoe, H. Gordon, S. Hollandsworth, X. Jiang, C. Johnson, N. Lawrence, J. Lean, J. Lerner, K. Lo, J. Logan, A. Luckett, M. P. McCormick, R. McPeters, R.
Miller, P. Minnis, I. Ramberran, G. Russell, P. Russell, P. Stone, I. Tegen, S. Thomas, L. Thomason, A. Thompson, J. Wilder, R. Willson, and J. Zawodny, J. Geophys. Res. 102, 25679, DOI: 10.1029/97JD01495 (1997).

${ }^{44}$ J. G. Freire, C. A. L. Pires, C. DaCamara, and J. A. C. Gallas (submitted). ${ }^{45}$ P. G. Lind, J. A. C. Gallas, and H. J. Herrmann, Phys. Rev. E 70, 056207 (2004); P. G. Lind, A. Nunes, and J. A. C. Gallas, Physica A 371, 100 (2006).

${ }^{46}$ A. A. Tsonis and P. J. Roebber, Physica D 333, 497 (2004); P. J. Roebber and A. A. Tsonis, J. Atmos. Sci. 62, 3818 (2005). 